\title{
Dynamic Reconfiguration of Structural and Functional Connectivity Across Core Neurocognitive Brain Networks with Development
}

\author{
Lucina Q. Uddin, ${ }^{1 \star}$ Kaustubh S. Supekar, ${ }^{1 \star}$ Srikanth Ryali, ${ }^{1}$ and Vinod Menon ${ }^{1,2,3}$ \\ Departments of ${ }^{1}$ Psychiatry and Behavioral Sciences, ${ }^{2}$ Neurology and Neurological Sciences and ${ }^{3}$ Program in Neuroscience, Stanford University School of \\ Medicine, Stanford, California 94305
}

Brain structural and functional development, throughout childhood and into adulthood, underlies the maturation of increasingly sophisticated cognitive abilities. High-level attentional and cognitive control processes rely on the integrity of, and dynamic interactions between, core neurocognitive networks. The right fronto-insular cortex (rFIC) is a critical component of a salience network (SN) that mediates interactions between large-scale brain networks involved in externally oriented attention [central executive network (CEN)] and internally oriented cognition [default mode network (DMN)]. How these systems reconfigure and mature with development is a critical question for cognitive neuroscience, with implications for neurodevelopmental pathologies affecting brain connectivity. Using functional and effective connectivity measures applied to fMRI data, we examine interactions within and between the SN, CEN, and DMN. We find that functional coupling between key network nodes is stronger in adults than in children, as are causal links emanating from the rFIC. Specifically, the causal influence of the rFIC on nodes of the SN and CEN was significantly greater in adults compared with children. Notably, these results were entirely replicated on an independent dataset of matched children and adults. Developmental changes in functional and effective connectivity were related to structural connectivity along these links. Diffusion tensor imaging tractography revealed increased structural integrity in adults compared with children along both within- and between-network pathways associated with the rFIC. These results suggest that structural and functional maturation of rFIC pathways is a critical component of the process by which human brain networks mature during development to support complex, flexible cognitive processes in adulthood.

\section{Introduction}

The human brain undergoes a protracted period of development during which widespread changes occur. The maturation of specific functional systems underlies the development of increasingly sophisticated cognitive functions from childhood to adulthood, including working memory, attention, and cognitive control (Bunge and Crone, 2009). These functions are implemented through interactions within and between large-scale brain networks (Mesulam, 1998; Bressler and Menon, 2010). Network analyses are increasingly used to assess global, interde-

Received Aug. 31, 2011; revised 0ct. 8, 2011; accepted Oct. 22, 2011.

Author contributions: V.M. designed research; L.Q.U., K.S.S., and S.R. performed research; K.S.S., S.R., and V.M. contributed unpublished reagents/analytic tools; L.Q.U., K.S.S., and S.R. analyzed data; L.Q.U., K.S.S., and V.M. wrote the paper.

This work was supported by National Institute of Mental Health Career Development Award K01MH092288 (L.Q.U.), National Institutes of Health Grants HD047520, HD059205, HD057610, and MH084164 (V.M.), and National Science Foundation Grant BCS/DRL 0449927 (V.M.). We thank Katie Karlsgodt for helpful comments on this manuscript, Leeza Kondos, Katherine Prater, and Jose Anguiano for assistance with data collection, and F. Xavier Castellanos, Bennett Leventhal, and Michael Milham for providing the Nathan Kline Institute/Rockland Sample (http://fcon_1000.projects.nitrc.org/indi/pro/nki.html).

*L.Q.U. and K.S.S. contributed equally to this work.

The authors declare no competing financial interests.

Correspondence should be addressed to Lucina Q. Uddin and Vinod Menon, 401 Quarry Road, Stanford, CA 94305-5719. E-mail: lucina@stanford.edu, menon@stanford.edu.

DOI:10.1523/JNEUROSCI.4465-11.2011

Copyright $\odot 2011$ the authors $\quad 0270-6474 / 11 / 3118578-12 \$ 15.00 / 0$ pendent properties of the developing brain and to understand dynamic maturation processes (Chu-Shore et al., 2011).

Some principles governing the development of gray and white matter have emerged. Structural neuroimaging has shown that while gray matter volume follows a regionally specific inverted U-shaped trajectory, white matter volume shows protracted increases with development (Lenroot and Giedd, 2006; Giedd and Rapoport, 2010). Diffusion tensor imaging (DTI) studies have shown that anisotropy increases and overall diffusion decreases with age (Cascio et al., 2007). How structural changes impact functional brain maturation is less well understood.

Understanding dynamic reconfiguration of brain networks between childhood and adulthood requires identifying changes in structural and functional connectivity during this period. In the adult brain, several canonical brain networks have been identified (Damoiseaux et al., 2006). Three of these can be considered core neurocognitive networks because of their critical roles in high-level cognition: (1) a frontoparietal central executive network (CEN) comprising the dorsolateral prefrontal cortex (DLPFC) and posterior parietal cortex (PPC), related to maintenance and manipulation of information and decision making in the context of goal-directed behavior; (2) a default mode network $(\mathrm{DMN})$, including the ventromedial prefrontal cortex (VMPFC) and posterior cingulate cortex (PCC), associated with internally oriented and social cognition; and (3) a salience network (SN) 
Table 1. Participant demographics

\begin{tabular}{lll}
\hline & Children $(n=23)$ & Adults $(n=22)$ \\
\hline Age & 7.95 years (range, 7-9 years) & 20.40 years (range, 19-22 years) \\
Gender & 10 males, 13 females & 11 males, 11 females \\
IQ & 112 (range, 88-137) & 112 (range, 97-137) \\
\hline
\end{tabular}

Participant groups did not differ on $\mathrm{IQ}$ or gender distribution.

with nodes in the right fronto-insular cortex (rFIC) and anterior cingulate cortex (ACC), involved in attention as well as interoceptive and affective processes (Sridharan et al., 2008). An important hypothesized function of the $\mathrm{SN}$ is to identify relevant internal and extrapersonal stimuli to guide behavior (Seeley et al., 2007). The rFIC node of the $\mathrm{SN}$ has been shown to initiate control signals that enable switching between the CEN and DMN in response to cognitive demands, acting as a "causal outflow hub" coordinating other large-scale networks (Sridharan et al., 2008; Menon and Uddin, 2010). In networks, hubs allow for increased levels of information flow between distant nodes, acting as integrators (Honey et al., 2007). A causal outflow hub is a region that has a high number of causal outflow connections and low number of causal inflow connections as assessed using Granger causality analyses (GCA).

Little is known about the neural systems supporting the development of these control processes. Here, we examine developmental changes in brain network interactions using multimodal imaging, combining functional and effective connectivity of intrinsic fMRI and DTI tractography. We hypothesized that we would find differences in causal outflow from network nodes between children and adults, with more dramatic changes with development detected in the rFIC, and that these changes would be further reflected in structural connectivity measures.

\section{Materials and Methods \\ Participants}

Twenty-three children and 22 IQ-matched adults participated in this study after providing written informed consent. For those children who were unable to give informed consent, written informed consent was obtained from their legal guardian. The study protocol was approved by the Stanford University Institutional Review Board. Children (10 males, 13 females) ranged in age from 7 to 9 years (mean age, 7.95 years), with an IQ range of 88-137 (mean IQ, 112). Adults (11 males, 11 females) ranged in age from 19 to 22 years (mean age, 20.4 years), with an IQ range of 97-137 (mean IQ, 112). All participants were recruited locally: children from local schools and adults from Stanford University and neighboring community colleges in the greater San Francisco Bay area (Table 1).

\section{Data acquisition}

\section{Functional MRI}

For the resting-state fMRI scan, subjects were instructed to keep their eyes closed and try not to move for the duration of the 8 min scan. Functional images were acquired on a 3 T GE Signa scanner (GE Healthcare) using a custom-built head coil. Head movement was minimized during scanning by a comfortable custom-built restraint. A total of 29 axial slices $(4.0 \mathrm{~mm}$ thickness, $0.5 \mathrm{~mm}$ skip) parallel to the anterior commissure-posterior commissure (AC-PC) line and covering the whole brain were imaged using a $\mathrm{T} 2^{\star}$-weighted gradient echo spiral inout pulse sequence (Glover and Law, 2001) with the following parameters: TR, $2000 \mathrm{~ms}$; TE, $30 \mathrm{~ms}$; flip angle, $80^{\circ}$; 1 interleave. The field of view was $20 \mathrm{~cm}$, and the matrix size was $64 \times 64$, providing an in-plane spatial resolution of $3.125 \mathrm{~mm}$. To reduce blurring and signal loss arising from field inhomogeneities, an automated high-order shimming method based on spiral acquisitions was used before acquiring functional MRI scans.

\section{Structural MRI}

For each subject, a high-resolution T1-weighted spoiled grass gradient recalled (SPGR) inversion recovery 3D MRI sequence was acquired to facilitate anatomical localization of functional and DTI data. The following parameters were used: TI, $300 \mathrm{~ms}$; TR, $8.4 \mathrm{~ms}$; TE, $1.8 \mathrm{~ms}$; flip angle, $15^{\circ} ; 22 \mathrm{~cm}$ field of view; 132 slices in coronal plane; $256 \times 192$ matrix; number of excitation, 2 ; acquired resolution, $1.5 \times 0.9 \times 1.1 \mathrm{~mm}$.

\section{DTI}

DTI data were obtained from 18 of the 23 children and 15 of 22 adults. The DTI pulse sequence was a diffusion-weighted single-shot spin-echo, echo planar imaging sequence (TE, $70.8 \mathrm{~ms}$; TR, $8.6 \mathrm{~s}$; field of view, 220 $\mathrm{mm}$; matrix size, $128 \times 128$; bandwidth, $\pm 110 \mathrm{kHz}$; partial $k$-space acquisition). We acquired 63 axial, 2-mm-thick slices (no skip) for two $b$ values: $b=0$ and $b=\sim 850 \mathrm{~s} / \mathrm{mm}^{2}$. The high $b$ value was obtained by applying gradients along 23 different diffusion directions. Two gradient axes were energized simultaneously to minimize TE. The polarity of the effective diffusion-weighting gradients was reversed for odd repetitions to reduce cross-terms between diffusion gradients and imaging and background gradients. Previous work of Jones (2004) suggests that measuring more diffusion directions is a more efficient way to reliably estimate diffusion tensors of arbitrary orientation. Although we were not able to obtain more diffusion directions in the current study, we were able to obtain four repeats, producing a signal-to-noise ratio that is sufficiently high to produce very reliable tensor estimates suitable for tractography.

\section{Data processing}

\section{Functional MRI}

Preprocessing. A linear shim correction was applied separately for each slice during reconstruction using a magnetic field map acquired automatically by the pulse sequence at the beginning of the scan (Glover and Lai, 1998). Functional MRI data were then analyzed using SPM5 analysis software (http://www.fil.ion.ucl.ac.uk/spm). Images were realigned to correct for motion, corrected for errors in slice timing, spatially transformed to standard stereotaxic space [based on the Montreal Neurologic Institute (MNI) coordinate system], resampled every $2 \mathrm{~mm}$ using sinc interpolation and smoothed with a $6 \mathrm{~mm}$ full-width half-maximum Gaussian kernel to decrease spatial noise before statistical analysis. Translational movement in millimeters $(x, y, z)$ and rotational motion in degrees ( pitch, roll, yaw) was calculated based on the SPM5 parameters for motion correction of the functional images in each subject. No participants had a range of movement $>3 \mathrm{~mm}$ translation or 3 degrees of rotation. Motion parameters did not differ between children and adults.

Independent component analysis. Each participant's smoothed, normalized images were concatenated across time to form a four-dimensional matrix using FSL 3.3 [for FMRIB Software Library (in which FMRIB is Functional MRI of the Brain), Oxford University, Oxford, UK]. This fourdimensional matrix was then analyzed with FSL 4.4 melodic independent component analysis (ICA) concatenated across participants. This analysis was limited to output only 25 components for the group. From these components, networks of interest—SN, CEN, and DMN—were selected for subsequent analyses using previously validated methods (Greicius et al., 2004). These components were then binarized using SPM5 to create templates for choosing network components for individuals.

Each participant's smoothed, normalized, four-dimensional matrix was analyzed with FSL melodic ICA version 12. The number of components output by ICA was determined automatically by the principal component analysis process of the melodic software. For adults, the number of ICA components generated ranged from 23 to 93 . For children, the number of ICA components generated ranged from 45 to 74 . The templates created above for the three networks were then applied to each participant's individual ICA components to select the "best-fit" network component. To do this, we developed a nonlinear template-matching procedure that involved taking the average $z$-score of voxels falling within the template minus the average $z$-score of voxels outside the template and selecting the component in which this difference (the goodness-of-fit) was the greatest. $z$-scores here reflect the degree to which the time series of a given voxel correlates with the times series corresponding to the specific ICA component. There were no 
differences between the child and adult groups with respect to goodness-offit scores to each of the templates (DMN, $p=0.15$; CEN, $p=0.10$; SN, $p=$ $0.54)$. The template-matching method allowed us to select each individual's $\mathrm{SN}, \mathrm{CEN}$, and DMN. A combined-group analysis was performed using the individual best-fit network components for the three networks. Individual $t$-statistic images from both groups were used to determine combined group-level statistical maps using a one-sample $t$ test as implemented in SPM5. The results were masked with a skull-stripped binary image of the standard MNI T1. Significant clusters were determined using a voxel-wise statistical height $(p<0.01)$ and extent $(p<0.01)$ threshold, corrected at the whole-brain level. These steps were undertaken to avoid bias and ensure that equal weight was given to networks identified in both children and adults because previous work suggests that, although resting-state networks are reliably and consistently identifiable in adults (Damoiseaux et al., 2006), they can be more variable in their presentation in children (de Bie et al., 2011).

Region-of-interest selection. We defined regions of interest (ROIs) in key nodes of the $\mathrm{SN}$, CEN, and DMN based on the peaks of the ICA clusters. ROIs were selected from respective combined-group ICA clusters (created as described above): in the rFIC and ACC (on the SN ICA map); in the rDLPFC and rPPC (on the CEN ICA map); and in the VMPFC and PCC (on the DMN ICA map). After selecting voxels with the highest $z$-scores within each cluster on the functional map, the final ROIs were constructed by drawing spheres with centers as the seed point and a radius of $8 \mathrm{~mm}$. Corresponding left-hemisphere ROIs were also created to explore laterality effects (Table 2). These ROI selection procedures are widely used in extant functional and effective connectivity studies (Fox et al., 2005; Fair et al., 2008; Kelly et al., 2009; Supekar et al., 2010).

Functional connectivity analysis. The regional resting-state fMRI time series was computed for each of the ROIs by averaging all the voxels within each region at each time point in the time series, resulting in 235 time points for each of the ROIs. These regional time series were temporally filtered using a bandpass filter $(0.0083 \mathrm{~Hz}<f<0.15 \mathrm{~Hz})$. We used partial correlation as a measure of strength of functional connectivity between the network brain regions. Partial correlation measures the degree of association between two regions, controlling for the effect of other regions, and has been widely used in task- and resting-state fMRI (Sun et al., 2004; Salvador et al., 2005; Liu et al., 2008; van den Heuvel et al., 2008; Supekar et al., 2010). As noted by van den Heuvel et al. (2008), this partial correlation approach has significant advantages over the pure correlation approach used in previous studies. In particular, our analysis allows the investigation of functional connectivity uncontaminated by activity in other nodes under investigation. To account for the non-normality of partial correlations, a Fisher's $r$-to- $z$ transform was applied.

Multivariate GCA. Multivariate GCA was performed in accordance with the methods of Seth (2010). First, the mean time course from each ROI was extracted for all subjects. Each time series was then detrended, and its temporal mean was removed. GCA was performed to test for causal influences between ROIs. The order of the vector autoregressive (VAR) model used for computation of the influence measure was selected using the Akaike information criterion. We proceeded to construct group-wise causal connectivity graphs from these raw $F$ values as described next. We performed statistical inferencing on the causal connections using nonparametric analyses. Empirical null distributions of influence terms ( $F$ values) and their differences were estimated nonparametrically by generating surrogate datasets under the null hypothesis that there are no causal interactions between the regions. To generate an instance of surrogate data, Fourier transform was applied to each from this analysis.
Table 2. Coordinates of SN, CEN, and DMN regions derived from ICA of resting-state fMRI data

\begin{tabular}{lllcr}
\hline Network & Region & BA & Peak MNI coordinates $(\mathrm{mm})$ & $Z$-score \\
\hline SN & FFIC & 47 & $39,23,-4$ & 11.66 \\
& IFIC & 47 & $-34,20,-8$ & 7.99 \\
& ACC & 24 & $6,24,32$ & 13.16 \\
CEN & rDLPFC & 9 & $46,20,44$ & 14.47 \\
& IDLPFC & 9 & $-46,20,44$ & 7.77 \\
& IPPC & 40 & $52,-52,50$ & 11.06 \\
& IPPC & 40 & $-40,-56,44$ & 11.12 \\
DMN & VMPFC & 11 & $-2,38,-12$ & 10.08 \\
& PCC & $23 / 30$ & $-6,-44,34$ & 10.36 \\
\hline
\end{tabular}

Figure 1. Identifying nodes of neurocognitive networks. SN, right CEN, and DMN identified from ICA of resting-state fMRI data. components. Network nodes for subsequent analyses were based on 8-mm-radius spheres created around peak voxels defined

regional time series, and the phase of the transformed signal was randomized. Inverse Fourier transform was then applied to generate one instance of surrogate data. This procedure ensures that the magnitude spectrum of the data is preserved while any causal interactions between various regions are eliminated. Test statistics were then computed by fitting the VAR model to the surrogate data. This procedure was repeated for several instances of surrogate data $(n=500)$ to obtain the null distribution of $F$ values and their differences. Those directed connections whose mean (across subjects in the group) was significantly different from the mean of the null ( $F$ value) distribution were identified using statistical tests and a stringent threshold $[p<$ 0.01 , false discovery rate (FDR) corrected]. The stringent threshold was chosen to avoid potentially spurious causal links introduced by the low temporal resolution and hemodynamic blurring in the fMRI signal. In addition, a difference of influence (doi) term, $\left(F_{x \rightarrow y}-F_{y \rightarrow x}\right)$, was used to assess links that showed a dominant direction of influence; the difference term further limits potentially spurious links caused by hemodynamic blurring (Roebroeck et al., 2005). Again, these dominant links were those wherein the mean of the doi term significantly differed from the empirically constructed null distribution ( $p<0.01$, FDR corrected for multiple comparisons). Between-group differences in the causal connectivity graphs were determined as follows. Differences in doi terms between the two groups were computed, for each link. The links that showed between-group changes in the strength of causal influence were those whose mean difference in the doi 

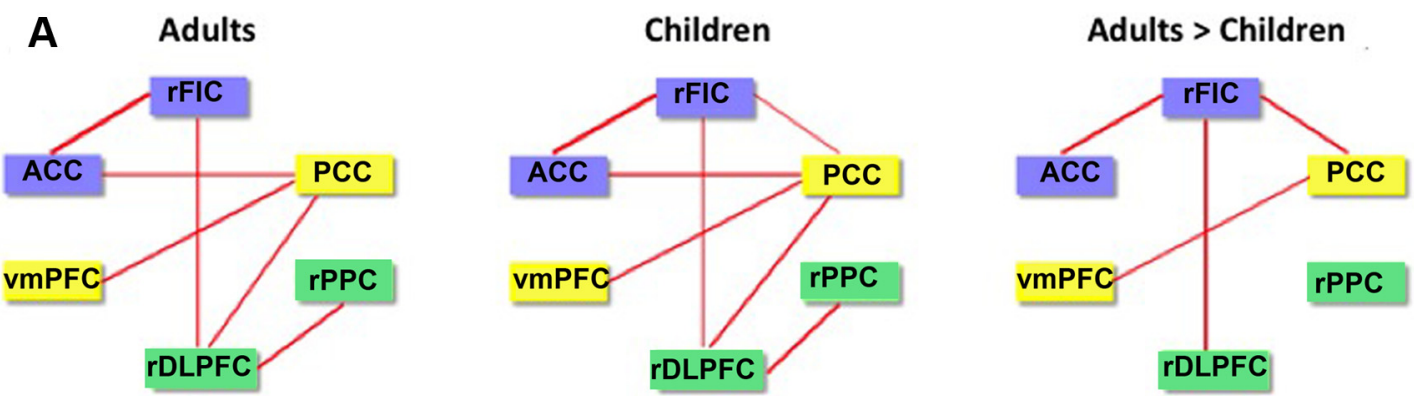

\begin{tabular}{|llllll|}
\hline \begin{tabular}{|lllll} 
Partial Correlation \\
(relative to max)
\end{tabular} & $\overline{100 \%}$ & $\overline{80 \%}$ & $\overline{60 \%}$ & $\overline{40 \%}$ & $\overline{20 \%}$ \\
\hline
\end{tabular}
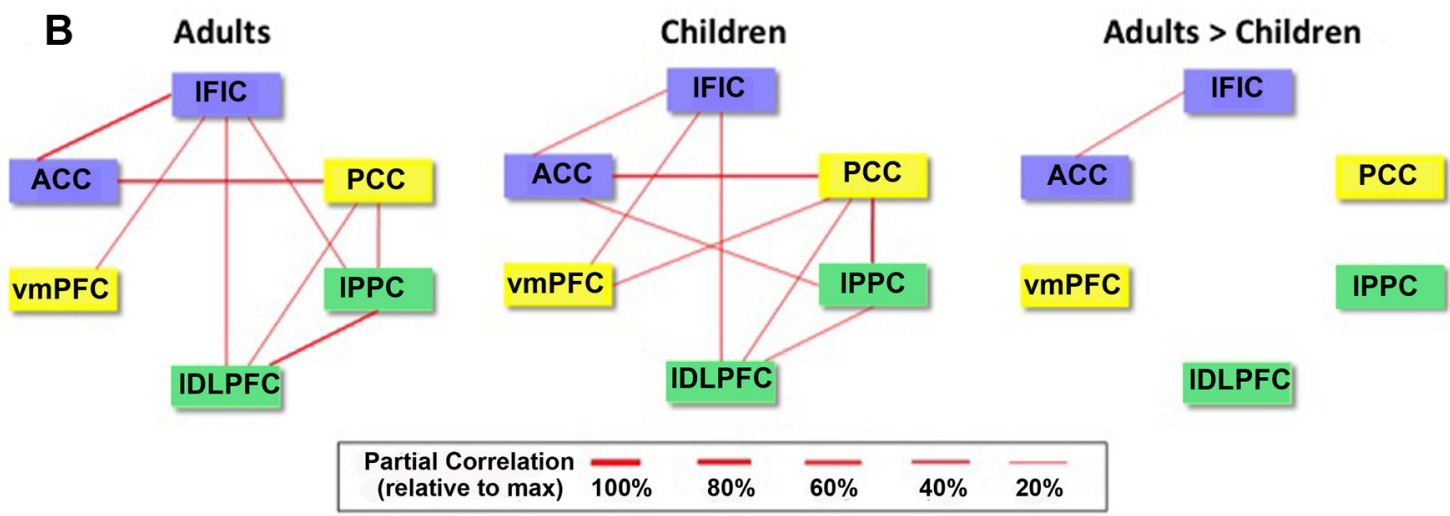

Figure 2. Functional connectivity between nodes of neurocognitive networks. $\boldsymbol{A}$, Instantaneous (undirected) functional connectivity, as measured by partial correlation, of the six key nodes of the SN (blue), CEN (green), and DMN (yellow) in adults and children and results of a two-sample $t$ test contrasting the functional connectivity in children versus adults ( $p<0.01$, FDR corrected). $\boldsymbol{B}$, Results from analysis of left-hemisphere ROls.

term significantly differed from empirically constructed null distributions of difference of doi terms ( $p<0.01$, FDR corrected).

Network analysis. We calculated the following metrics to further characterize the networks in children and adults: (1) out-degree, number of causal outflow connections from a node in the network to any other node; (2) in-degree, number of causal in-flow connections to a node in the network from any other node; and (3) (out-in) degree, difference between out degree and in degree, a measure of the net causal outflow from a node.

Replication analyses. We identified from the publicly available Nathan Kline Institute dataset (http://fcon_1000.projects.nitrc.org/indi/pro/nki. html) a group of 22 adult participants in the age range of 19-22 years who did not differ in mean age, gender distribution, or IQ from our adult dataset. Because there were not a sufficient number of 7- to 9-year-old participants available from the Nathan Kline Institute dataset, we identified from our own database an independent group of 23 children matched on age, gender, and IQ to the original sample. Using these new datasets for independent verification, we conducted identical functional and effective connectivity analyses as in the original dataset.

\section{DTI}

DTI data were preprocessed using a custom program based on normalized mutual information that removed eddy current distortion effects and determined a constrained nonrigid image registration of all the diffusion images (Bammer et al., 2002). The six elements of the diffusion tensor were determined by multivariate regression (Basser, 1995; Basser and Pierpaoli, 1996). For each subject, the non-diffusion-weighted ( $b=$ 0 ) images were coregistered to the T1-weighted 3D SPGR anatomical images using a mutual information $3 \mathrm{D}$ rigid-body coregistration algorithm from SPM5. Several anatomical landmarks, including the AC, the $\mathrm{PC}$, and the midsagittal plane, were identified by hand on the T1 images. With these landmarks, we computed a rigid-body transform from the native image space to the conventional AC-PC-aligned space. The DTI data were then resampled to this AC-PC-aligned space with $2 \mathrm{~mm}$ isotropic voxels using a spline-based tensor interpolation algorithm $(\mathrm{Pa}-$ jevic et al., 2002), taking care to rotate the tensors to preserve their orientation with respect to the anatomy (Alexander et al., 2001). The T1 images were resampled to AC-PC-aligned space with $1 \mathrm{~mm}$ isotropic voxels. We confirmed by visual inspection of each dataset that this coregistration technique aligns the DTI and T1 images to within 1-2 $\mathrm{mm}$ in the brain ROIs.

Tractography. Using custom DTI analysis software (available for download at http://sirl.stanford.edu/software/), the tractography procedure was initiated by whole-brain fiber tracking in native space that produced many fiber paths. To ensure that we had obtained high-quality data suitable for tractography from each participant, we validated our data by following a procedure recommended by Wakana et al. (2007) for identifying major fiber tracts. Only participants with high-quality data as determined by this validation procedure were included in subsequent analyses.

First, for each participant, we computed the total number of streamlines by creating subject-specific white matter masks. For each voxel in a white matter mask, fiber tracking was initiated using the voxel as the seed. Starting from the initial seed, fiber paths were traced using a streamline algorithm in both directions along the principal diffusion axis. Path tracing proceeded until fractional anisotropy (FA) fell below 0.15 or until the minimum angle between the current and previous path segments was $>30^{\circ}$. We report the number of successful streamlines (fiber paths) across the whole brain for both child and adult groups and acknowledge that the changes in structural connectivity we report along tracts of interest may be influenced by more global changes with development.

We used ROIs defined from the Automated Anatomical Labeling (AAL) atlas as sources and targets for these analyses to ensure that the regions were sufficiently large to enable reliable tractography estimates (Jones and Cercignani, 2010). In principle, it would be desirable to conduct both functional and structural connectivity analyses using the same 
A Adults

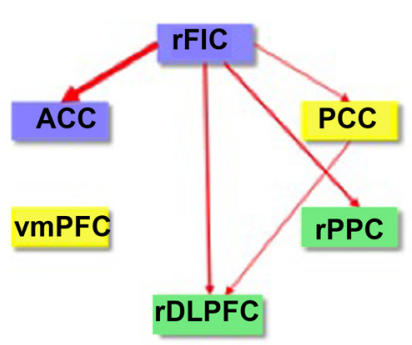

Children

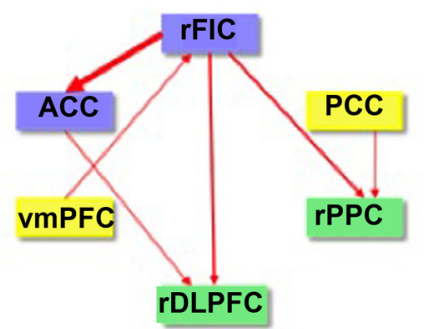

Adults > Children

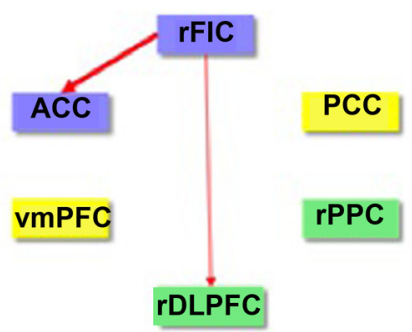

\begin{tabular}{|cccccc|}
\hline $\begin{array}{c}\text { Directed Influence } \\
\text { (relative to max) }\end{array}$ & $\mathbf{1 0 0 \%}$ & $\overrightarrow{80 \%}$ & $\overrightarrow{60 \%}$ & $\overrightarrow{40 \%}$ & $\overrightarrow{20 \%}$ \\
\hline
\end{tabular}
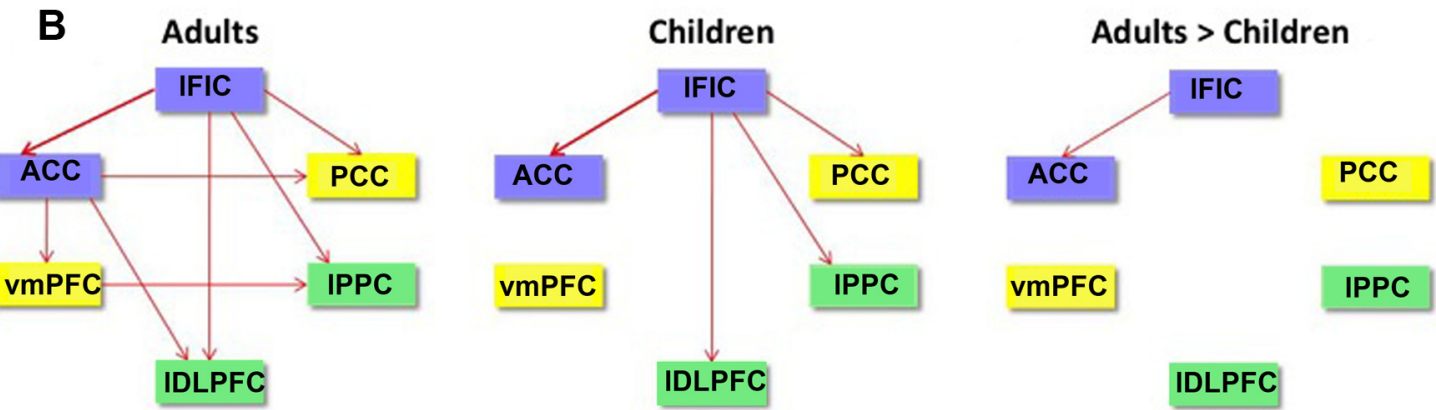

\begin{tabular}{|cccccc|}
\begin{tabular}{|c|c|c|} 
Directed Influence \\
(relative to max)
\end{tabular} & $\overrightarrow{100 \%}$ & $\overrightarrow{80 \%}$ & $\overrightarrow{60 \%}$ & $\overrightarrow{40 \%}$ & $\overrightarrow{20 \%}$ \\
\hline
\end{tabular}

Figure 3. Effective connectivity between nodes of neurocognitive networks. $A$, GCA of the six key nodes of the SN (blue), CEN (green), and DMN (yellow) in adults and children and results of a two-sample $t$ test contrasting directed causal network interactions in children versus adults ( $p<0.01$, FDR corrected). $\boldsymbol{B}$, Results from analysis of left-hemisphere ROls.

ROIs. However, the ROIs we used for the resting-state fMRI analyses were relatively small ( $8 \mathrm{~mm}$ radius). This was to ensure anatomical specificity and to avoid potentially including information from the time series of the neighboring regions (Smith et al., 2011). For the tractography, however, we have found both in the current study and in previous work (Supekar et al., 2010; Uddin et al., 2010b) that larger ROIs are necessary when examining structural connections. Jones also reports that use of small ROIs and subtle differences in ROI placement can have a large effect on the mean structural connectivity values derived from the ROI because of the rapidly changing values in FA maps between neighboring tissues and suggests that the best strategy for positioning ROIs varies depending on the shape, the size, and the location of the region (Jones and Cercignani, 2010). We therefore chose to use larger, anatomically defined ROIs as provided by the AAL atlas for the structural connectivity analyses to avoid these issues. Furthermore, the primary aim of the current study was not to link the statistical relations between functional and structural connectivity across large numbers of ROIs (as in the study by Hagmann et al., 2010) but rather to examine developmental differences in the configuration and connectivity of core neurocognitive networks. Our structural connectivity analyses were conducted as follow-up analyses to explore whether and to what extent the observed functional connectivity differences were related to structural connectivity differences. Importantly, our approach allows us to provide more detailed information about localization and integrity of white matter pathways associated with the rFIC.

The spatially normalized insula, ACC, and DLPFC ROIs obtained from the AAL atlas (Tzourio-Mazoyer et al., 2002) were warped back to each individual brain to be used for DTI tractography analyses. This was done by applying the inverse of the spatial normalization transformation. Because fiber tracking becomes unreliable in gray matter, we ensured that our ROIs extended 2-3 $\mathrm{mm}$ into the white matter. Tracts that did not end in or pass through both ROIs were discarded. Each fiber tract was estimated using a deterministic streamline tracking algorithm (Conturo et al., 1999; Mori et al., 1999; Basser et al., 2000) with a fourth-order Runge-Kutta path integra- tion method (Press, 2002) and $1 \mathrm{~mm}$ fixed step size. A continuous tensor field was estimated using trilinear interpolation of the tensor elements. Starting from the initial seed point, fiber paths were traced in both directions along the principal diffusion axis. Path tracing proceeded until the FA fell below 0.15 or until the minimum angle between the current and previous path segments was $>30^{\circ}$. To limit the number of false positives, fibers that were anatomically implausible were identified visually and removed for the rFIC-ACC connections. The rFIC-rDLPFC tracts were further constrained by those labeled as belonging to the fronto-occipital fasciculus using the Johns Hopkins University white matter tractography atlas (http://www.fmrib.ox.ac.uk/fsl/data/atlas-descriptions.html).

Structural connectivity. For each subject, the mean FA and fiber density connecting the rFIC to the ACC and the rFIC to the rDLPFC were measured, in native space. The mean FA was computed by averaging FA values along the fiber tracts of interest. FA is a measure of organization of the underlying white matter (Beaulieu, 2002). Fiber density is the number of fibers per unit surface and approximates the axonal number interconnecting the ROIs. Both FA and fiber density have been used previously to quantify structural connectivity, particularly to characterize white matter integrity (Hagmann et al., 2008; van den Heuvel et al., 2008). In this study, we used mean FA and fiber density as measures of the integrity of the fiber tracts of interest. For each subject, the density of the fibers connecting each source to target ROI pair was measured. The density of fibers connecting two regions $u$ and $v$ was computed as follows:

$$
\text { Fiber density }(u, v)=\frac{2}{\mathrm{~S} u+\mathrm{S} v} \sum_{f \in \mathrm{F}(u, v)} \frac{1}{l(f)}
$$

where $F(u, v)$ is a set of all the fibers $f$ connecting $u$ and $v, l(f)$ is the length of the individual fiber, and $S u$ and $S v$ are sizes of the ROIs (Hagmann et al., 2008). 

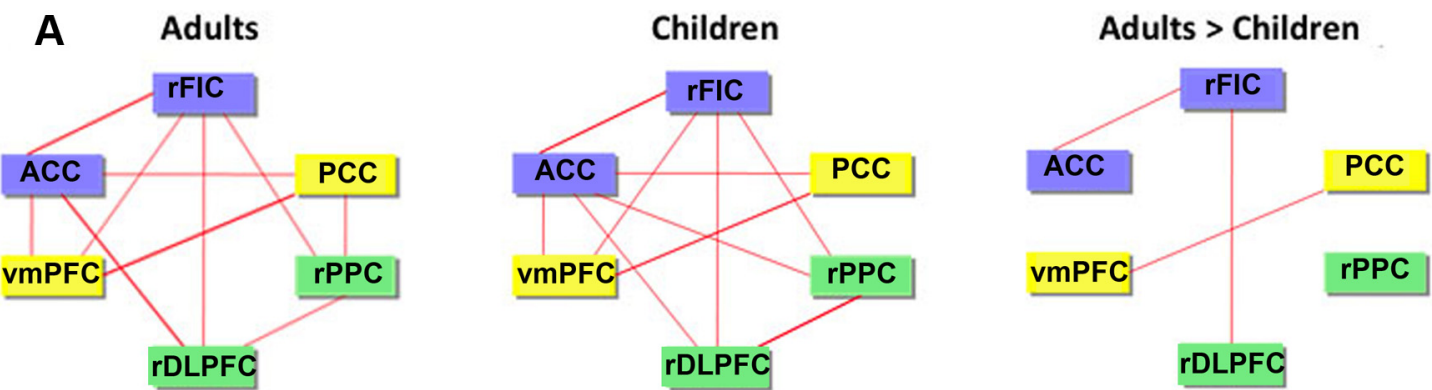

\begin{tabular}{|c|c|c|c|c|c|}
\hline $\begin{array}{l}\text { Partial Correlation } \\
\text { (relative to max) }\end{array}$ & $\overline{100 \%}$ & $\overline{80 \%}$ & $\overline{60 \%}$ & $\overline{40 \%}$ & $20 \%$ \\
\hline
\end{tabular}
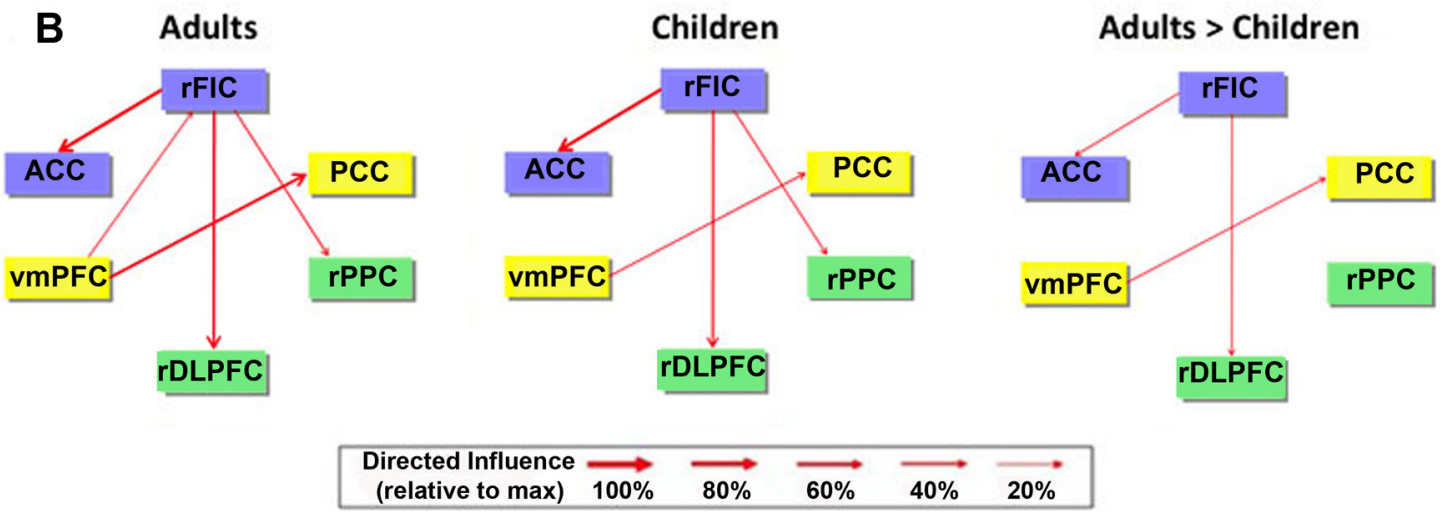

Figure 4. Replication analysis of functional and effective connectivity between nodes of neurocognitive networks. Using data from the publicly available NKI dataset (http:// fcon_1000.projects.nitrc.org/indi/pro/nki.html), functional and effective connectivity analyses were conducted, replicating the original findings. A, Functional connectivity (partial correlation) between network nodes in children and adults. $\boldsymbol{B}$, Effective connectivity (GCA) between network nodes in children and adults.
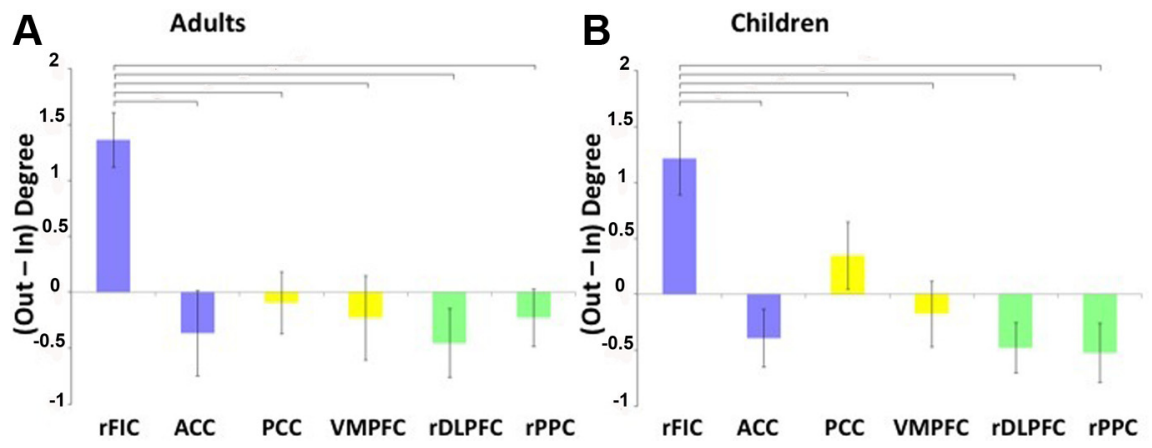

Figure 5. Net outflow of effective connectivity between nodes of neurocognitive networks. Net causal outflow (out-in degree) in the key nodes of the SN (blue), DMN (yellow), and CEN (green) are shown for adults $(\boldsymbol{A})$ and children $(\boldsymbol{B})$. In both groups, the rFIC had significantly higher net causal outflow than the ACC, PCC, VMPFC, $\mathrm{DDLPFC}$, and $\operatorname{PPPC}(p<0.01$, FDR corrected).
ROIs showed greater functional connectivity in children compared with adults $(p<$ 0.01 , FDR corrected). These group differences in functional connectivity were entirely replicated when we conducted this analysis on an independent matched dataset of children and adults (downloaded from http://fcon_1000.projects.nitrc.org/indi/ pro/nki.html) (see Fig. 4A).

Examining the left-hemisphere counterparts of these network nodes, we found that the only link showing a group difference in partial correlation (adult $>$ children) was the left FIC (lFIC)-ACC connection $(p<0.01$, FDR corrected $)$ (Fig. 2B).

\section{Results}

Comparison of functional connectivity between network nodes in children versus adults

To assess developmental effects on the strength of functional coupling between the network nodes, we computed instantaneous partial correlations between pairs of ROIs (Fig. 1). We found that rFIC connectivity with ACC, rDLPFC, and PCC was stronger in adults compared with children. In addition, connectivity between PCC and VMPFC was stronger in adults compared with children $(p<0.01$, FDR corrected) (Fig. $2 A)$, as reported previously (Supekar et al., 2010). Thus, both within-network (rFICACC, PCC-VMPFC) and between-network (rFIC-rDLPFC, rFICPCC) connectivity patterns were stronger in the older cohort. No
Comparison of effective connectivity between network nodes in children versus adults

We used multivariate GCA to investigate causal interactions between the six network nodes. GCA detects causal interactions between brain regions by assessing the predictability of signal changes in one brain region based on the time course of responses in another brain region (Goebel et al., 2003). Although there are some concerns that systematic differences across brain regions in hemodynamic lag can potentially lead to spurious estimations of causality (Smith et al., 2011), recent analyses suggest that, when applied at the group level, GCA has good control over spurious results (Roebroeck et al., 2011; Schippers et al., 2011). Our detailed simulations (Ryali et al., 2011) suggest that GCA is able to 
recover causal network structure despite the presence of HRF delay confounds. Because other definitions and models of causality exist in the literature (Friston, 2009), we specify that our effective connectivity results should be interpreted as Granger causality results.

We performed GCA using a multivariate model on the time courses extracted from each of the ROIs. In adults, GCA revealed significant direct causal influences from the rFIC to the ACC, rDLPFC, rPPC, and PCC. In children, GCA revealed causal influences from the rFIC to these same regions except the PCC. Quantitative comparison of group differences in the strength of causal influences revealed that the strength of interactions from the rFIC to the ACC and from the rFIC to the rDLPFC was significantly greater in adults compared with children $(p<0.01$, FDR corrected), as shown in Figure $3 A$. Thus, both within-network (rFIC-ACC) and between-network (rFIC-rDLPFC) causal influences were stronger in the older cohort. No links showed greater causal influence in children compared with adults.

These group differences in effective connectivity were entirely replicated when we conducted this analysis again on an independent matched dataset of children and adults, with the exception that an additional group difference emerged in the replication dataset (adult $>$ children VMPFC-PCC causal influence) (Fig. 4B).

Examining the left-hemisphere counterparts of these network nodes, we found that the only link showing a group difference in GCA (adult $>$ children) was the lFIC-ACC connection $(p<$ 0.01 , FDR corrected) (Fig. 3B). Thus, the lFIC is not as strong a driver of network dynamics as is the $\mathrm{rFIC}$, as reported previously (Sridharan et al., 2008).

We calculated the net outflow of causal interactions in both groups to replicate previous findings suggesting that the rFIC acts as a causal outflow hub and to explore for the first time whether this holds true in children. Figure 5 depicts the results of a network analysis computing the out-in degree for each node of the $\mathrm{SN}, \mathrm{DMN}$, and CEN. In both children and adults, the rFIC had significantly higher net causal outflow than the ACC, PCC, VMPFC, rDLPFC, and rPPC ( $p<0.01$, FDR corrected $)$.

\section{Comparison of structural connectivity between network nodes in children versus adults}

We used deterministic tractography to examine potential differences in structural connectivity between children and adults. For children, the mean number of streamlines was 55,768 \pm 5263 , and, for adults, the mean was $63,609 \pm 6114$. Thus, adults had a significantly higher number of whole-brain streamlines (structural connectivity) compared with children $(p<0.001)$. This finding of generally increased whole-brain structural connectivity with age is consistent with previous literature (Barnea-Goraly et al., 2005; Supekar et al., 2009).

To further explore potential factors underlying the functional and effective connectivity results, we examined structural connectivity between the nodes that showed both differential func- tional and effective connectivity between children and adults. White matter tracts along the uncinate fasciculus connecting rFIC to ACC were detected in 11 of 15 adults (73\%) and 9 of 18 children (50\%) examined. A one-tailed Mann-Whitney nonparametric test was conducted to evaluate the hypothesis that adults would show greater structural connectivity than children. We found that, compared with adults, children showed lower mean fiber density in the rFIC-ACC tracts (effect size, $0.63 ; p=$ 0.05 ). This group difference was present even after correcting for global structural connectivity differences between children and adults. The FA measure showed a similar trend, although the group difference was not significant (effect size, $0.46 ; p=0.1$ ) (Figs. 6, 7).

Tracts between the rFIC and rDLPFC were detected in 14 of 15 adults (93\%) and 12 of 18 (67\%) children examined. The mean fiber density along the fronto-occipital fasciculus connecting rFICrDLPFC showed a strong trend toward being lower in children compared with adults (effect size, $0.33 ; p=0.07$ ), an effect that again was still present after correcting for global structural connectivity differences between children and adults. The FA measure showed significant group differences (effect size, $0.82 ; p=0.01$ ) (Figs. 8,9). Thus, pathways showing both reduced functional and effective connectivity in children compared with adults also showed absent or reduced structural connectivity as measured with DTI.

To explore relationships between functional and structural connectivity, we computed correlations between functional connectivity (as measured by normalized partial correlation) and structural connectivity (as measured by FA) in both the child and adult groups for both the rFIC-ACC and rFIC-rDLPFC links. For the rFIC-rDLPFC link, we found a significant correlation between functional connectivity and structural connectivity in 

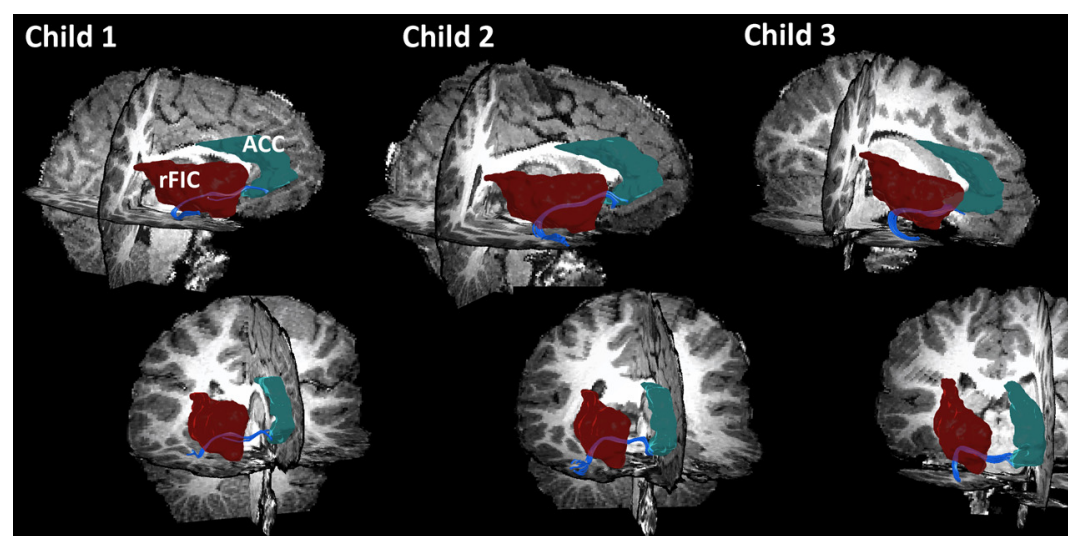

\section{Child 4}

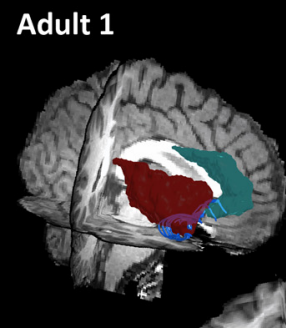

\section{Adult 2}
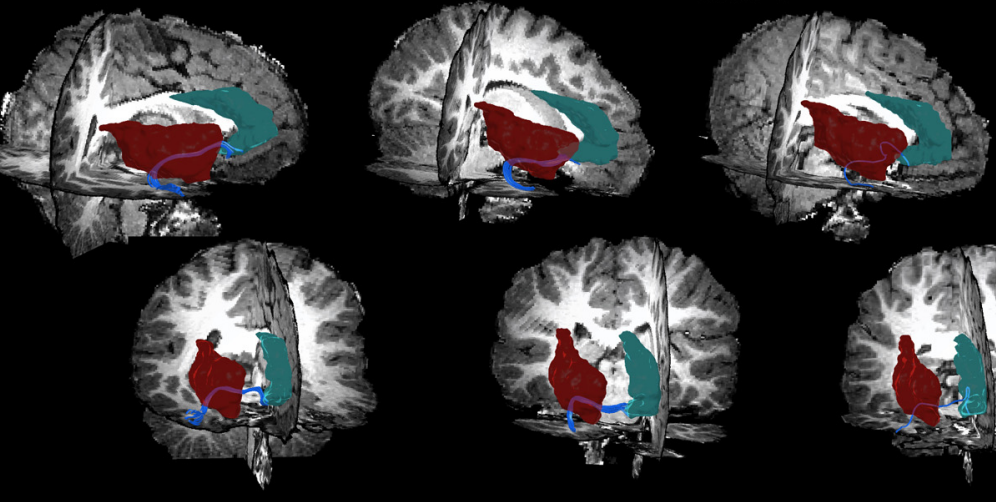

Adult 3

\section{Adult 4}
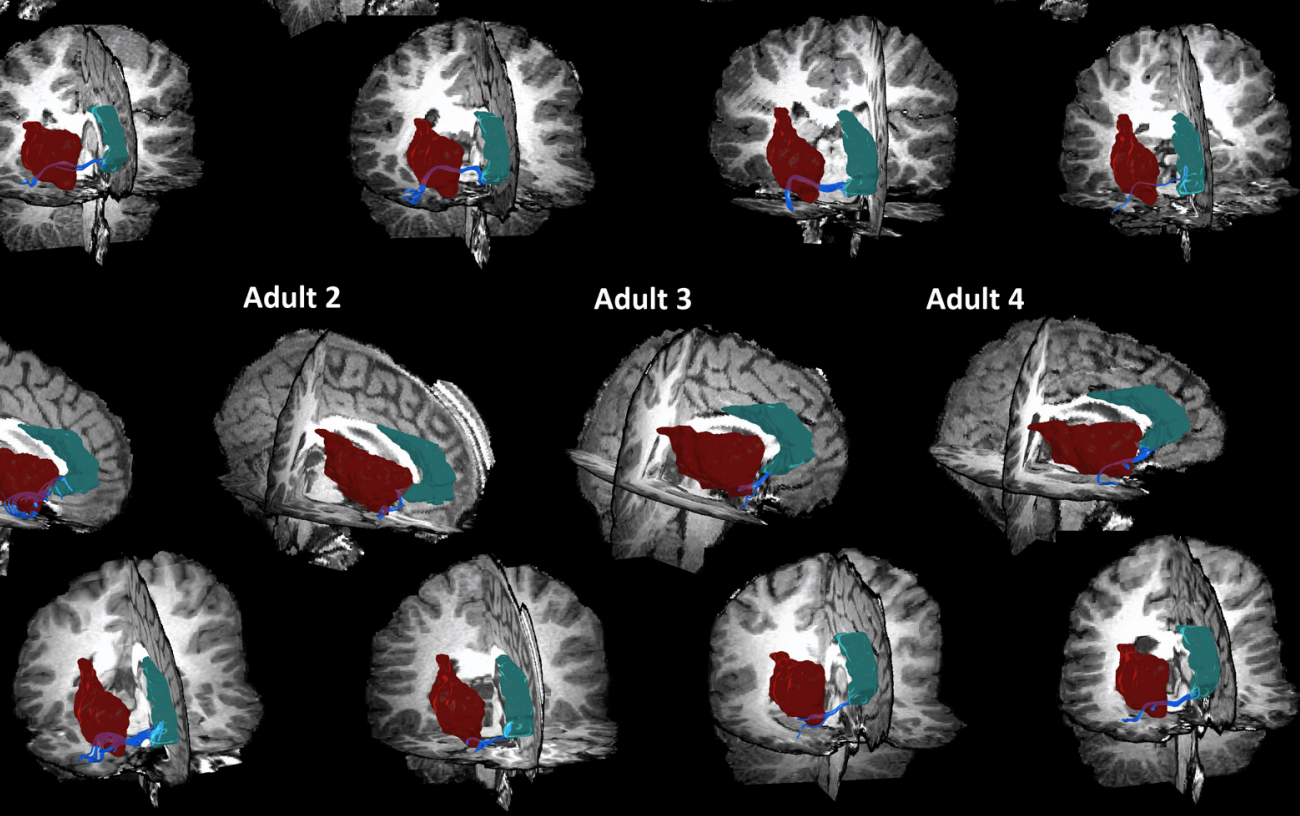

Figure 7. Structural connectivity between nodes of SN: $r F I C-A C C$ in eight individuals. DTI tractography results show that tracts along the uncinate fasciculus can be reliably identified in individual subjects. Tracts were detected in 11 of 15 adults (73\%) and 9 of 18 children (50\%). Four children and adults are shown. The first row shows a sagittal slice viewed from the right, whereas the second row shows a coronal slice viewed anteriorly.

the adult group $(r=0.81, p=0.0004)$ but not in the child group $(r=-0.18, p=0.58)$ (Fig. 10). No significant correlations were found between functional and structural connectivity for the adult group or the child group for the rFIC-ACC link.

\section{Discussion}

The human brain undergoes dramatic maturational changes from childhood to adulthood, enabling increasingly sophisticated cognitive abilities. Recent approaches seek to investigate this maturation in the context of interconnected networks (Somerville and Casey, 2010; Uddin et al., 2010a). Here we examined developmental changes of core neurocognitive networks involved in salience detection ( $\mathrm{SN}$ ), externally oriented attention (CEN), and internally oriented cognition (DMN) using functional, effective, and structural connectivity measures. Functional connectivity between several pairs of nodes, both within and between networks, was significantly greater in adults compared with children. Specifically, rFIC-ACC and PCC-VMPFC (within-network) and rFIC-rDLPFC and rFIC-PCC (betweennetwork) coupling was greater in adults compared with children. The strength of directed interactions for a subset of these node pairs (rFIC-ACC and rFIC-rDLPFC) was greater in adults compared with children, a finding confirmed in an independent dataset. Thus, the causal influence of the rFIC on other brain areas becomes stronger with development, even though the rFIC can already be identified as a causal outflow hub in children. Structural connectivity along both rFIC-rDLPFC and rFIC-ACC tracts was lower in children compared with adults.
Development of functional and effective connectivity across core neurocognitive brain networks

Although global functional brain organization is similar in children and adults, at the subnetwork level, connectivity undergoes significant reorganization with development (Fair et al., 2009; Supekar et al., 2009). Adults have weaker short-range functional connectivity and stronger long-range functional connectivity than children (Kelly et al., 2009), and the organization of functional brain networks shifts from local connectivity to a more distributed architecture with development (Fair et al., 2007; Supekar et al., 2009). Here we show, for the first time, regional specificity in the development of strengthened within- and between-network relationships. Critically, rFIC connectivity displayed the most dramatic developmental effects. IFIC connectivity patterns, although similar, were weaker and less robust. We have recently proposed that the rFIC is in a unique position to facilitate bottom-up access to the attentional and working memory resources of the brain, functioning to detect salient environmental or endogenous events and initiate switching between the DMN and CEN (Menon and Uddin, 2010). The rFIC is situated at the interface of the cognitive, homeostatic, and affective systems of the brain, providing a link between stimulus-driven processing and regions involved in monitoring the internal milieu (Craig, 2009). The fact that this region shows both increased integration with other components of the SN (ACC) and increased causal outflow to other control network nodes (DLPFC) suggests that it may play a critical role in the development of functions attributed to the $\mathrm{SN}$, 
including attention allocation and cognitive control (Menon and Uddin, 2010). While a previous study demonstrated age-related increases in the strength of functional connectivity between rFIC-ACC (Fair et al., 2007), there have been no previous investigations of the development of effective and structural connectivity along this pathway. Strikingly, the rFIC was the only node that underwent within- and between-network functional and effective connectivity changes across the age groups examined. This finding was entirely replicated in an independent dataset of matched adults and children, despite differences in scanner model and acquisition parameters.

Previous studies have shown developmental changes in the strength of functional connectivity along the anterior and posterior nodes of the DMN (Supekar et al., 2010; Gordon et al., 2011). The current study addresses the complex issue of how connections both within and between key networks mature with development in ways that can produce flexible and adaptive behavior.

Traditionally, ventrolateral prefrontal cortex has been associated with cognitive control (Petrides, 2005). However, recent work has shown a primary and critical role for the rFIC in control processes. A meta-analysis of neuroimaging studies of motor inhibition and reflexive reorienting found that the right anterior insula/frontal operculum was the most active region across studies (Levy and Wagner, 2011). Transcranial magnetic stimulation evidence suggests that the frontal operculum causally exerts influence over posterior areas in the context of selective attention (Higo et al., 2011). Causal links from the rFIC to dorsal attention and DMNs across experimental states (Gao and Lin, 2011), as well as causal links emanating from the rFIC during resting states (Deshpande et al., 2011) have also been reported, supporting the idea that rFIC regulates other networks for effective resource allocation and maintenance of appropriate task-relevant behavioral states. What we have termed the salience network is also referred to as the cingulo-opercular network (Dosenbach et al., 2007), but it is generally agreed on that an independent network comprising nodes in the rFIC and ACC can be identified across both resting (Seeley et al., 2007) and task states (Medford and Critchley, 2010).

In a previous study using ICA and "causal density" estimates to characterize functional networks in participants ranging in age from 12 to 30 years, Stevens et al. (2009) reported that mutual influences among networks decreased with age, reflecting stronger within-network connectivity and more efficient between-network influences with development. They also found age-related reductions in the strength of interaction between executive control circuits and the DMN and suggest that more segregated functioning of these networks may allow greater processing flexibility (Stevens et al., 2009). The current work examines causal interactions between network nodes rather than entire networks (Smith et al., 2011), providing insight into maturation of specific connections with development.

\section{Development of structural connectivity across core neurocognitive brain networks}

To explore structural changes related to the observed functional changes, we conducted targeted DTI tractography analyses. The strength of functional connectivity between two nodes is positively correlated with structural connectivity (Hagmann et al., 2010), although functional connectivity can exist independent of structural connectivity (Uddin et al., 2008; Honey et al., 2009). Although there have been reports combining tractography and functional connectivity to study development (Supekar et al., 2009; Hagmann et al., 2010), no previous studies have used fiber tracking to examine the trajectory and integrity of rFIC-related tracts as they develop between childhood and adulthood. Tracts connecting the rFIC to rDLPFC run along the fronto-occipital fasciculus, involved in visuospatial processing and attention, whereas those connecting the rFIC to ACC run along the uncinate fasciculus, a ventral limbic pathway critical for processing novel information and enabling emotion-cognition interaction (Schmahmann et al., 2007; Mori et al., 2009). For both rFICrDLPFC and rFIC-ACC pathways, functional connectivity increases were paralleled by structural connectivity increases with development. Although it is unlikely that new axonal fiber tracts are created between the ages examined, it is known that structural maturation of white matter continues during adolescence (Paus, 2010). Increased fiber density and FA may reflect increased myelination (Rademacher et al., 1999), changes in axonal diameter, and the relative alignment of individual axons and their packing density (Paus, 2010). 


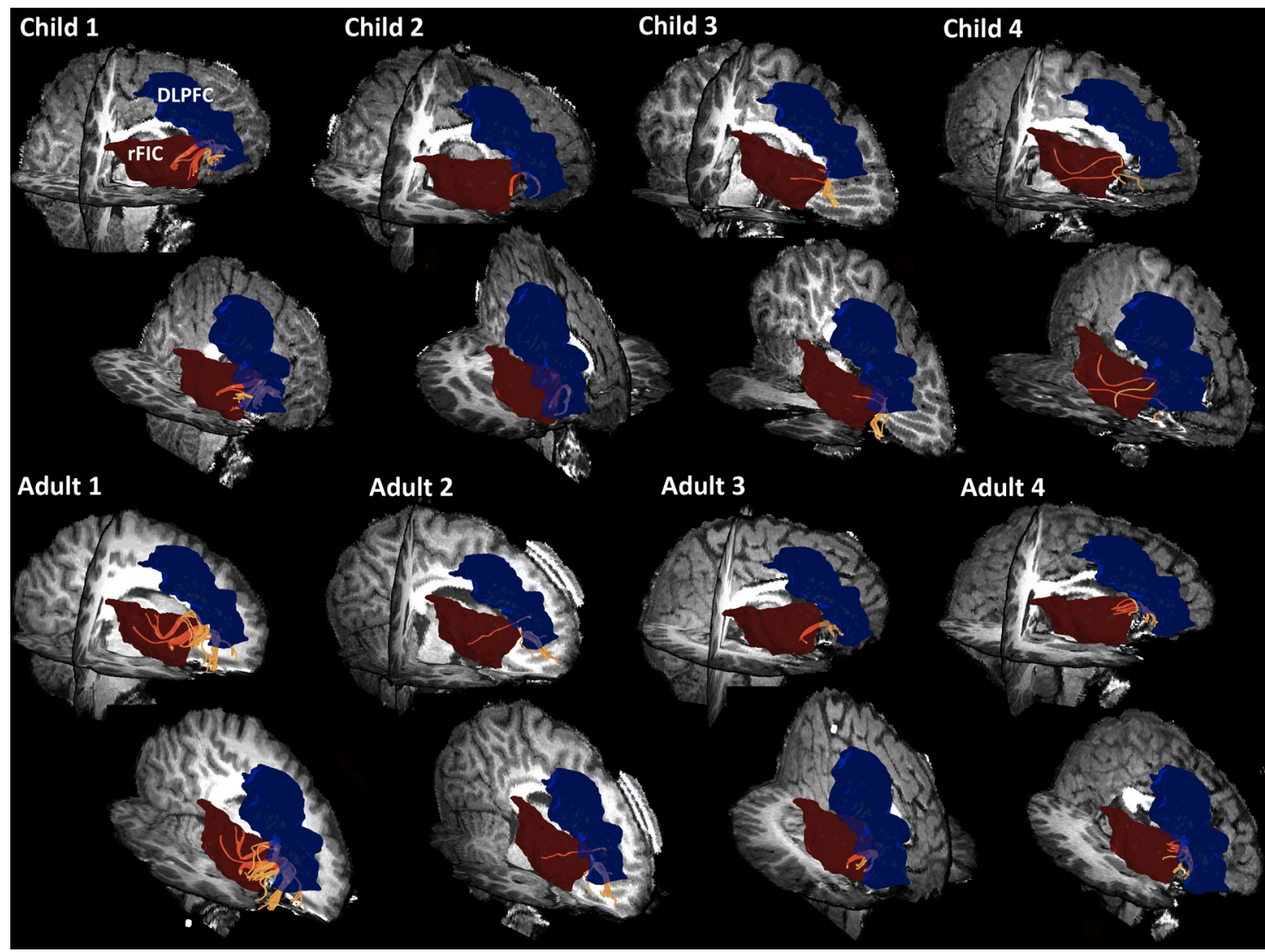

Figure 9. Structural connectivity between nodes of SN and CEN: rFIC-rDLPFC in eight individuals. DTI tractography results show that tracts along the fronto-occipital fasciculus can be reliably identified in individual subjects. Tracts were detected in 14 of 15 adults (93\%) and 12 of $18(67 \%)$ children. Four children and adults are shown. The first row shows a sagittal slice viewed from the right, whereas the second row show a sagittal slice viewed dorsally.

rFIC-rDLPFC Structure-Function Correlation

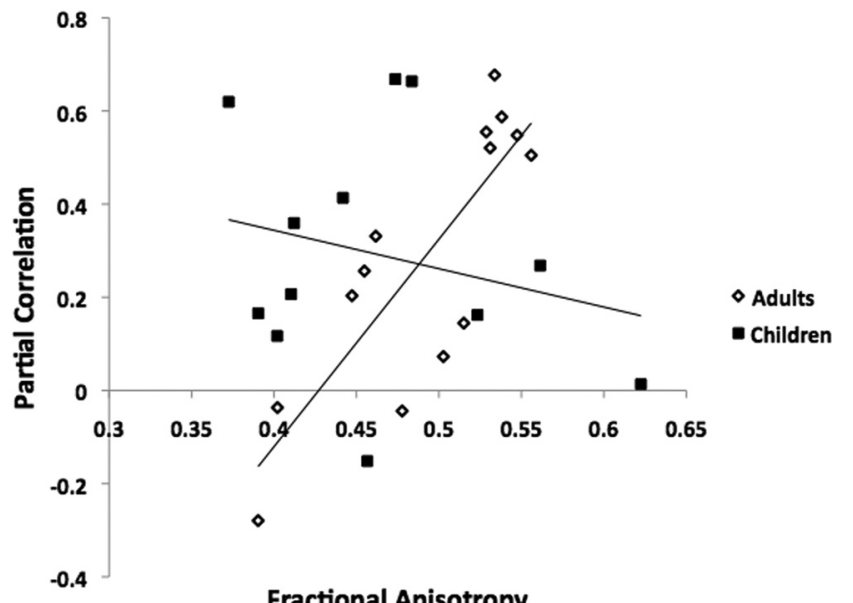

Figure 10. Relationship between functional connectivity and structural connectivity. In adults, there was a significant correlation between functional connectivity (as measured by normalized partial correlation) and structural connectivity (as measured by FA) for the rFICrDLPFC link ( $r=0.81, p=0.0004)$. There were no other significant correlations between functional and structural connectivity in the adults or children.

To our knowledge, no previous study has examined structural connectivity of the rFIC-rDLPFC pathway. We found a significant correlation between functional and structural connectivity in this pathway in adults but not in children. This finding is in line with a small but growing body of literature showing that struc- ture-function relationships become more stable and strengthen with development (Hagmann et al., 2010; Supekar et al., 2010; Gordon et al., 2011). We did not observe a similar dissociation for the rFIC-ACC tract, raising the possibility that the development of specific structural and functional connections of the rFIC may follow independent developmental trajectories.

Only one previous study examined both structural and functional connectivity of the rFIC-ACC pathway, reporting that structural links are detectable in some (38\%) adults who demonstrate functional connectivity between these regions (van den Heuvel et al., 2009). Our study is the first to identify the ventral uncinate fasciculus pathway linking the rFIC-ACC nodes of the $\mathrm{SN}$ and to demonstrate it is relatively immature in 7- to 9-yearold children.

\section{Implications for neurodevelopmental disorders of connectivity}

The rFIC pathways identified here are of particular interest in neurodevelopmental disorders involving disrupted connectivity, such as autism spectrum disorders (ASDs) (Geschwind and Levitt, 2007). In neurotypical adults, functional connectivity between the anterior insula and ACC is related to scores on a measure of social responsiveness (Di Martino et al., 2009). Participants with ASD show decreased functional connectivity between the rFIC and ACC (Ebisch et al., 2011). Thus, atypical development of this critical pathway may contribute to altered social attentional processes associated with autism, an open question for future research (Uddin and Menon, 2009). 


\section{Conclusions}

The rFIC has been shown to play a causal role in switching between the CEN and the DMN in adults (Sridharan et al., 2008). This process is likely involved in switching attention to salient exogenous and endogenous events to produce appropriate behavioral responses. We found that, in 7- to 9-year-old children, the influence of the rFIC on nodes within the SN and CEN is weaker than in adults, possibly as a result of immature anatomical connectivity between the rFIC and other network nodes. Notably, these findings were also observed in an independent dataset of matched children and adults.

In the current study, we conducted a hypothesis-driven analysis of three specific neurocognitive networks. However, ICA of resting-state fMRI data often reveal multiple functional networks, including the three examined in the current study and several others associated with sensory, motor, and other systems (De Luca et al., 2006; Stevens et al., 2009). Future work examining all of these networks may reveal an even more detailed picture of changes in network configuration and rFIC connectivity during development. Our findings have important implications for understanding the development of increasingly sophisticated cognitive control abilities subserved by this dynamic multi-network system (Menon and Uddin, 2010) and point to potential loci of dysfunction that may be targeted for additional investigation in studies of neurodevelopmental disorders involving the rFIC, including autism.

\section{References}

Alexander DC, Pierpaoli C, Basser PJ, Gee JC (2001) Spatial transformations of diffusion tensor magnetic resonance images. IEEE Trans Med Imaging 20:1131-1139.

Bammer R, Auer M, Keeling SL, Augustin M, Stables LA, Prokesch RW, Stollberger R, Moseley ME, Fazekas F (2002) Diffusion tensor imaging using single-shot SENSE-EPI. Magn Reson Med 48:128-136.

Barnea-Goraly N, Menon V, Eckert M, Tamm L, Bammer R, Karchemskiy A, Dant CC, Reiss AL (2005) White matter development during childhood and adolescence: a cross-sectional diffusion tensor imaging study. Cereb Cortex 15:1848-1854.

Basser PJ (1995) Inferring microstructural features and the physiological state of tissues from diffusion-weighted images. NMR Biomed 8:333-344.

Basser PJ, Pierpaoli C (1996) Microstructural and physiological features of tissues elucidated by quantitative-diffusion-tensor MRI. J Magn Reson B 111:209-219.

Basser PJ, Pajevic S, Pierpaoli C, Duda J, Aldroubi A (2000) In vivo fiber tractography using DT-MRI data. Magn Reson Med 44:625-632.

Beaulieu C (2002) The basis of anisotropic water diffusion in the nervous system - a technical review. NMR Biomed 15:435-455.

Bressler SL, Menon V (2010) Large-scale brain networks in cognition: emerging methods and principles. Trends Cogn Sci 14:277-290.

Bunge SA, Crone EA (2009) Neural correlates of the development of cognitive control. In: Neuroimaging in developmental clinical neuroscience (Rumsey JM, Ernst M, eds). Cambridge, UK: Cambridge UP.

Cascio CJ, Gerig G, Piven J (2007) Diffusion tensor imaging: application to the study of the developing brain. J Am Acad Child Adolesc Psychiatry 46:213-223.

Chu-Shore CJ, Kramer MA, Bianchi MT, Caviness VS, Cash SS (2011) Network analysis: applications for the developing brain. J Child Neurol 26:488-500.

Conturo TE, Lori NF, Cull TS, Akbudak E, Snyder AZ, Shimony JS, McKinstry RC, Burton H, Raichle ME (1999) Tracking neuronal fiber pathways in the living human brain. Proc Natl Acad Sci USA 96:10422-10427.

Craig AD (2009) How do you feel—now? The anterior insula and human awareness. Nat Rev Neurosci 10:59-70.

Damoiseaux JS, Rombouts SA, Barkhof F, Scheltens P, Stam CJ, Smith SM, Beckmann CF (2006) Consistent resting-state networks across healthy subjects. Proc Natl Acad Sci U S A 103:13848-13853.

de Bie HM, Boersma M, Adriaanse S, Veltman DJ, Wink AM, Roosendaal SD,
Barkhof F, Stam CJ, Oostrom KJ, Delemarre-van de Waal HA, SanzArigita EJ (2011) Resting-state networks in awake five- to eight-year old children. Hum Brain Mapp. Advance online publication. Retrieved November 7, 2011. doi:10.1002/hbm.21280.

De Luca M, Beckmann CF, De Stefano N, Matthews PM, Smith SM (2006) fMRI resting state networks define distinct modes of long-distance interactions in the human brain. Neuroimage 29:1359-1367.

Deshpande G, Santhanam P, Hu X (2011) Instantaneous and causal connectivity in resting state brain networks derived from functional MRI data. Neuroimage 54:1043-1052.

Di Martino A, Shehzad Z, Kelly C, Roy AK, Gee DG, Uddin LQ, Gotimer K, Klein DF, Castellanos FX, Milham MP (2009) Relationship between cingulo-insular functional connectivity and autistic traits in neurotypical adults. Am J Psychiatry 166:891-899.

Dosenbach NU, Fair DA, Miezin FM, Cohen AL, Wenger KK, Dosenbach RA, Fox MD, Snyder AZ, Vincent JL, Raichle ME, Schlaggar BL, Petersen SE (2007) Distinct brain networks for adaptive and stable task control in humans. Proc Natl Acad Sci U S A 104:11073-11078.

Ebisch SJ, Gallese V, Willems RM, Mantini D, Groen WB, Romani GL, Buitelaar JK, Bekkering H (2011) Altered intrinsic functional connectivity of anterior and posterior insula regions in high-functioning participants with autism spectrum disorder. Hum Brain Mapp 32:1013-1028.

Fair DA, Dosenbach NU, Church JA, Cohen AL, Brahmbhatt S, Miezin FM, Barch DM, Raichle ME, Petersen SE, Schlaggar BL (2007) Development of distinct control networks through segregation and integration. Proc Natl Acad Sci U S A 104:13507-13512.

Fair DA, Cohen AL, Dosenbach NU, Church JA, Miezin FM, Barch DM, Raichle ME, Petersen SE, Schlaggar BL (2008) The maturing architecture of the brain's default network. Proc Natl Acad Sci USA 105:4028-4032.

Fair DA, Cohen AL, Power JD, Dosenbach NU, Church JA, Miezin FM, Schlaggar BL, Petersen SE (2009) Functional brain networks develop from a "local to distributed" organization. PLoS Comput Biol 5:e1000381.

Fox MD, Snyder AZ, Vincent JL, Corbetta M, Van Essen DC, Raichle ME (2005) The human brain is intrinsically organized into dynamic, anticorrelated functional networks. Proc Natl Acad Sci U S A 102:9673-9678.

Friston K (2009) Causal modelling and brain connectivity in functional magnetic resonance imaging. PLoS Biol 7:e33.

Gao W, Lin W (2011) Frontal parietal control network regulates the anticorrelated default and dorsal attention networks. Hum Brain Mapp. Advance online publication. Retrieved November 7, 2011. doi:10.1002/hbm. 21204 .

Geschwind DH, Levitt P (2007) Autism spectrum disorders: developmental disconnection syndromes. Curr Opin Neurobiol 17:103-111.

Giedd JN, Rapoport JL (2010) Structural MRI of pediatric brain development: what have we learned and where are we going? Neuron 67:728 -734.

Glover GH, Lai S (1998) Self-navigated spiral fMRI: interleaved versus single-shot. Magn Reson Med 39:361-368.

Glover GH, Law CS (2001) Spiral-in/out BOLD fMRI for increased SNR and reduced susceptibility artifacts. Magn Reson Med 46:515-522.

Goebel R, Roebroeck A, Kim DS, Formisano E (2003) Investigating directed cortical interactions in time-resolved fMRI data using vector autoregressive modeling and Granger causality mapping. Magn Reson Imaging 21:1251-1261.

Gordon EM, Lee PS, Maisog JM, Foss-Feig J, Billington ME, Vanmeter J, Vaidya CJ (2011) Strength of default mode resting-state connectivity relates to white matter integrity in children. Dev Sci 14:738-751.

Greicius MD, Srivastava G, Reiss AL, Menon V (2004) Default-mode network activity distinguishes Alzheimer's disease from healthy aging: evidence from functional MRI. Proc Natl Acad Sci U S A 101:4637-4642.

Hagmann P, Cammoun L, Gigandet X, Meuli R, Honey CJ, Wedeen VJ, Sporns O (2008) Mapping the structural core of human cerebral cortex. PLoS Biol 6:e159.

Hagmann P, Sporns O, Madan N, Cammoun L, Pienaar R, Wedeen VJ, Meuli R, Thiran JP, Grant PE (2010) White matter maturation reshapes structural connectivity in the late developing human brain. Proc Natl Acad Sci U S A 107:19067-19072.

Higo T, Mars RB, Boorman ED, Buch ER, Rushworth MF (2011) Distributed and causal influence of frontal operculum in task control. Proc Natl Acad Sci U S A 108:4230-4235.

Honey CJ, Kötter R, Breakspear M, Sporns O (2007) Network structure of 
cerebral cortex shapes functional connectivity on multiple time scales. Proc Natl Acad Sci U S A 104:10240-10245.

Honey CJ, Sporns O, Cammoun L, Gigandet X, Thiran JP, Meuli R, Hagmann P (2009) Predicting human resting-state functional connectivity from structural connectivity. Proc Natl Acad Sci U S A 106:2035-2040.

Jones DK (2004) The effect of gradient sampling schemes on measures derived from diffusion tensor MRI: a Monte Carlo study. Magn Reson Med 51:807-815.

Jones DK, Cercignani M (2010) Twenty-five pitfalls in the analysis of diffusion MRI data. NMR Biomed 23:803-820.

Kelly AM, Di Martino A, Uddin LQ, Shehzad Z, Gee DG, Reiss PT, Margulies DS, Castellanos FX, Milham MP (2009) Development of anterior cingulate functional connectivity from late childhood to early adulthood. Cereb Cortex 19:640-657.

Lenroot RK, Giedd JN (2006) Brain development in children and adolescents: insights from anatomical magnetic resonance imaging. Neurosci Biobehav Rev 30:718-729.

Levy BJ, Wagner AD (2011) Cognitive control and right ventrolateral prefrontal cortex: reflexive reorienting, motor inhibition, and action updating. Ann NY Acad Sci 1224:40-62.

Liu Y, Liang M, Zhou Y, He Y, Hao Y, Song M, Yu C, Liu H, Liu Z, Jiang T (2008) Disrupted small-world networks in schizophrenia. Brain 131:945-961.

Medford N, Critchley HD (2010) Conjoint activity of anterior insular and anterior cingulate cortex: awareness and response. Brain Struct Funct 214:535-549.

Menon V, Uddin LQ (2010) Saliency, switching, attention and control: a network model of insula function. Brain Struct Funct 214:655-667.

Mesulam MM (1998) From sensation to cognition. Brain 121:1013-1052.

Mori S, Crain BJ, Chacko VP, van Zijl PC (1999) Three-dimensional tracking of axonal projections in the brain by magnetic resonance imaging. Ann Neurol 45:265-269.

Mori S, Oishi K, Faria AV (2009) White matter atlases based on diffusion tensor imaging. Curr Opin Neurol 22:362-369.

Pajevic S, Aldroubi A, Basser PJ (2002) A continuous tensor field approximation of discrete DT-MRI data for extracting microstructural and architectural features of tissue. J Magn Reson 154:85-100.

Paus T (2010) Growth of white matter in the adolescent brain: myelin or axon? Brain Cogn 72:26-35.

Petrides M (2005) Lateral prefrontal cortex: architectonic and functional organization. Philos Trans R Soc Lond B Biol Sci 360:781-795.

Press WH (2002) Numerical recipes in $\mathrm{C}++$ : the art of scientific computing, Ed 2. Cambridge, UK: Cambridge UP.

Rademacher J, Engelbrecht V, Bürgel U, Freund H, Zilles K (1999) Measuring in vivo myelination of human white matter fiber tracts with magnetization transfer MR. Neuroimage 9:393-406.

Roebroeck A, Formisano E, Goebel R (2005) Mapping directed influence over the brain using Granger causality and fMRI. Neuroimage 25: $230-242$.

Roebroeck A, Formisano E, Goebel R (2011) The identification of interacting networks in the brain using fMRI: model selection, causality and deconvolution. Neuroimage 58:296-302.

Ryali S, Supekar K, Chen T, Menon V (2011) Multivariate dynamical systems models for estimating causal interactions in fMRI. Neuroimage 54:807-823.

Salvador R, Suckling J, Coleman MR, Pickard JD, Menon D, Bullmore E (2005) Neurophysiological architecture of functional magnetic resonance images of human brain. Cereb Cortex 15:1332-1342.

Schippers MB, Renken R, Keysers C (2011) The effect of intra- and inter- subject variability of hemodynamic responses on group level Granger causality analyses. Neuroimage 57:22-36.

Schmahmann JD, Pandya DN, Wang R, Dai G, D’Arceuil HE, de Crespigny AJ, Wedeen VJ (2007) Association fibre pathways of the brain: parallel observations from diffusion spectrum imaging and autoradiography. Brain 130:630-653.

Seeley WW, Menon V, Schatzberg AF, Keller J, Glover GH, Kenna H, Reiss AL, Greicius MD (2007) Dissociable intrinsic connectivity networks for salience processing and executive control. J Neurosci 27:2349-2356.

Seth AK (2010) A MATLAB toolbox for Granger causal connectivity analysis. J Neurosci Methods 186:262-273.

Smith SM, Miller KL, Salimi-Khorshidi G, Webster M, Beckmann CF, Nichols TE, Ramsey JD, Woolrich MW (2011) Network modelling methods for FMRI. Neuroimage 54:875-891.

Somerville LH, Casey BJ (2010) Developmental neurobiology of cognitive control and motivational systems. Curr Opin Neurobiol 20:236-241.

Sridharan D, Levitin DJ, Menon V (2008) A critical role for the right frontoinsular cortex in switching between central-executive and default-mode networks. Proc Natl Acad Sci U S A 105:12569-12574.

Stevens MC, Pearlson GD, Calhoun VD (2009) Changes in the interaction of resting-state neural networks from adolescence to adulthood. Hum Brain Mapp 30:2356-2366.

Sun FT, Miller LM, D’Esposito M (2004) Measuring interregional functional connectivity using coherence and partial coherence analyses of fMRI data. Neuroimage 21:647-658.

Supekar K, Musen M, Menon V (2009) Development of large-scale functional brain networks in children. PLoS Biol 7:e1000157.

Supekar K, Uddin LQ, Prater K, Amin H, Greicius MD, Menon V (2010) Development of functional and structural connectivity within the default mode network in young children. Neuroimage 52:290-301.

Tzourio-Mazoyer N, Landeau B, Papathanassiou D, Crivello F, Etard O, Delcroix N, Mazoyer B, Joliot M (2002) Automated anatomical labeling of activations in SPM using a macroscopic anatomical parcellation of the MNI MRI single-subject brain. Neuroimage 15:273-289.

Uddin LQ, Menon V (2009) The anterior insula in autism: underconnected and under-examined. Neurosci Biobehav Rev 33:1198-1203.

Uddin LQ, Mooshagian E, Zaidel E, Scheres A, Margulies DS, Kelly AM, Shehzad Z, Adelstein JS, Castellanos FX, Biswal BB, Milham MP (2008) Residual functional connectivity in the split-brain revealed with restingstate functional MRI. Neuroreport 19:703-709.

Uddin LQ, Supekar K, Menon V (2010a) Typical and atypical development of functional human brain networks: insights from resting-state FMRI. Front Syst Neurosci 4:21.

Uddin LQ, Supekar K, Amin H, Rykhlevskaia E, Nguyen DA, Greicius MD, Menon V (2010b) Dissociable connectivity within human angular gyrus and intraparietal sulcus: evidence from functional and structural connectivity. Cereb Cortex 20:2636-2646.

van den Heuvel M, Mandl R, Luigjes J, Hulshoff Pol H (2008) Microstructural organization of the cingulum tract and the level of default mode functional connectivity. J Neurosci 28:10844-10851.

van den Heuvel MP, Mandl RC, Kahn RS, Hulshoff Pol HE (2009) Functionally linked resting-state networks reflect the underlying structural connectivity architecture of the human brain. Hum Brain Mapp 30:3127-3141.

Wakana S, Caprihan A, Panzenboeck MM, Fallon JH, Perry M, Gollub RL, Hua K, Zhang J, Jiang H, Dubey P, Blitz A, van Zijl P, Mori S (2007) Reproducibility of quantitative tractography methods applied to cerebral white matter. Neuroimage 36:630-644. 